\title{
La radioterapia en el cáncer de próstata localmente avanzado tras estadiaje ganglionar con linfadenectomía
}

\author{
Barbagelata López A, Ponce Díaz-Reixa JL, Romero Selas E, Gómez Veiga F, \\ Fernández Rosado E, Gonzalez Martín M.
}

Complejo Hospitalario Juan Canalejo. La Coruña.

Actas Urol Esp. 2006;30(9):856-865

\begin{abstract}
RESUMEN
LA RADIOTERAPIA EN EL CÁNCER DE PRÓSTATA LOCALMENTE AVANZADO TRAS ESTADIAJE GANGLIONAR CON LINFADENECTOMÍA

Introducción y objetivos: El cáncer de próstata localmente avanzado supone una condición de alto riesgo de progresión bioquímica post-tratamiento debido a la situación límite que representa. Nos planteamos analizar los factores pronósticos clínicos en función de la probabilidad de progresión tras emplear un tratamiento con fuente radioterapeútica externa en pacientes con este tipo de tumores.

Material y métodos: Revisamos retrospectivamente una serie de 128 pacientes sometidos a linfadenectomía pélvica de estadiaje como método de estadiaje previo a la realización de el tratamiento radioterápico. Empleamos las curvas de Kaplan-Meier para estudiar la probabilidad de progresión, el test de rangos logarítmicos para la demostración de posibles diferencias estadísticamente significativas y el modelo de riesgos proporcionales de Cox para estudiar los posibles factores pronósticos implicados.

Resultados: La probabilidad de no progresión a 5 años ha sido del 49,93\%; a pesar de apreciar diferencias importantes en los grupos estratificados por las variables predictivas empleadas (PSA total, gleason de biopsia, estadio patológico y \% de cilindros afectos en biopsia), ninguna de ellas alcanzó significación estadística, siendo el nivel de PSA t el más próximo a la misma.

Conclusiones: La radioterapia externa representa una alternativa válida en el tratamiento del cáncer de próstata localmente avanzado con un tolerable índice de secundarismos. Debe emplearse en tratamiento combinado junto a la hormonoterapia. Parece que el empleo de unas dosis más altas de radiación, posible de manera segura gracias a la radioterapia conformada 3D, permiten alcanzar una mayor eficacia. Es probable que el indicador clínico con mayores implicaciones pronósticas sea el nivel de PSA total.
\end{abstract}

Palabras clave : Cancer de próstata. Radioterapia. Tratamiento.

\begin{abstract}
EXTERNAL BEAM RADIOTHERAPY ON LOCALLY ADVANCED PROSTATE CARCINOMA FOLLOWING ILIAC STAGING LYMPHADENECTOMY

Introduction and objectives: Locally advanced prostate cancer supposes a high risk condition of post-treatment progression due to the limit situation that represents. Our purpose was to analyze prognoses factors in function of progression probability after using a treatment with external source radiotherapy on patients with this kind of tumors.

Material and methods: We retrospectively reviewed a set of 128 patients submitted to pelvic staging limphadenectomy prior to accomplish an external radiotherapeutic treatment. We employed the Kaplan-Meier curves to study the probability of progression, logarithmic ranks test were used for detection of possible statistically significant differences and proportional risks Cox model was employed to study possible risk factors of progression (employing astro criteria).

Results: 5 years freedom probability from progression was of 49,93\%; in spite of appreciating important differences in the groups stratified by the predictive variables used (total PSA, gleason of pathological biopsy, clinical stage and \% of cores affection on biopsy), none of them reached statistical meaning, being the level of total PSA the closest to it.

Conclusions: The external radiotherapeuthic treatment represents a valid alternative in the treatment of locally advanced prostate cancer, with a tolerable index of secondaries. It must be used combined with hormonotherapy. It seems that the use of higher radiation doses, in a safer way thanks to 3D conformed radiotherapy, allows to improve the results. The most powerful clinical predictor of evolution must be the total PSA.
\end{abstract}

Key words: Prostate cancer. Radiotherapy. Treatment. 
$\mathrm{E}$

cáncer de próstata es el tumor urológico que con más frecuencia se presenta en el varón, apreciándose en la Unión Europea una incidencia de 85.000 nuevos casos/año. En el ámbito de la Unión y en lo que se refiere a mortalidad se sitúa por detrás del cáncer colo-rectal y con escaso margen se sitúa por delante del cáncer de pulmón.

En España, el estudio realizado en la comunidad de Madrid, muestra una incidencia bruta de 100 casos/100.000 hombres. Y la distribución por estadios de los mismos es: $75 \%$ enfermedad localizada, $12,5 \%$ enfermedad metastática, $12,5 \%$ enfermedad localizada avanzada ${ }^{1}$.

No existe un consenso en la definición del concepto de cáncer de próstata localmente avanzado, y así los diferentes artículos de la literatura y las recomendaciones de las diferentes sociedades científicas varian en su acotación, referida al sistema de TNM de estadiaje, que oscila desde el T2b hasta T4 en cuanto al grado de infiltración glandular y desde el NO al N2 en cuanto al grado de afectación ganglionar. La inclusión de afectación ganglionar bajo este epígrafe supone una perversión, porque la localización primaria es la próstata. Nuestra referencia comunitaria, la Sociedad Europea de Urología en sus guías de actuación práctica lo define como: T3-T4, NO/Nx, MO/Mx y esta es la acepción que nosotros tomamos en consideración ${ }^{2}$.

Es un tipo de tumores especialmente delicados en su manejo porque sus condiciones oncológicas los sitúan en la frontera entre la enfermedad confinada, potencialmente curable con un gesto terapéutico, y la enfermedad extendida; siendo por tanto el estadiaje particularmente importante en su definición para poder predecir la evolución, por el salto cualitativo que supone un infraestadiaje en estos casos.

Dentro de las alternativas terapéuticas se encuentran: la prostatectomía radical, la radioterapia externa, el tratamiento hormonal y las terapias combinadas. La elección de ellas dependerá tanto de la esperanza de vida del paciente, como de sus preferencias y de la experiencia del grupo de trabajo que afronte la decisión terapéutica. Dentro de la comunidad urológica la alternativa que la literatura recoge como más empleada para estos casos es la Radioterapia Externa.
Presentando como base racional de utilización la mayor cobertura tisular alcanzable con esta técnica, por incluir en el campo terapéutico tejidos periglandulares.

\section{MATERIAL Y MÉTODOS}

Realizamos un análisis retrospectivo de los pacientes sometidos a tratamiento radioterapéutico con fuente externa, previamente estadiados mediante linfadenectomía pélvica, en nuestro centro en el periodo comprendido entre noviembre de 1995 y julio del 2003.

Los pacientes fueron sometidos a biopsia de próstata por la presencia de una exploración rectal anómala, por una determinación de PSA $t>4$, o con PSA $t<4$ si el \% de PSA libre era inferior al $25 \%$. La biopsia transrectal ecodirigida se realizó empleando un ecógrafo Phillips con una sonda de $6 \mathrm{Mhz}$ con visión biplanar simultánea, realizando las tomas de biopsia con agujas de punción $18 \mathrm{G}$.

Se realizó un protocolo randomizado de biopsias, tomando un número de cilindros variable entre 6 y 10, en función de criterios científicos evolutivos tomados en cuenta por la unidad de patología prostática y de las peculiaridades clínicas de los pacientes (nivel de PSA, anticoagulación,....).

Al diagnóstico se les informó en la sala de consulta de las alternativas terapéuticas aconsejadas y en aquellos que decidieron someterse al tratamiento con Radioterapia Externa se procedió a la realización de un estadiaje completo incluyendo gammagrafía ósea y linfadenectomía pélvica, rechazando a aquellos con afectación ganglionar.

Se les pautó bloqueo androgénico, previo a la realización del tratamiento radioterápico, que, se llevó a cabo en un centro colaborador especializado, mediante una técnica tradicional hasta 2002 para adherirse posteriormente a las técnicas de conformación 3D. Manteniendo el tratamiento hormonal hasta la revisión post-Rt que se fecha en torno al mes post-tratamiento.

En el seguimiento de estos pacientes, debido a que se presumía que la enfermedad estaba localizada en la próstata por la ausencia de afectación ganglionar, el objetivo era alcanzar el control local de su enfermedad, y, para ello, se mantuvieron los criterios de progresión bioquímica 
recomendados por la ASTRO (elevación de tres determinaciones consecutivas una vez alcanzado el nadir, fijando el punto temporal de progresión a medio camino entre la primera elevación y la determinación inmediatamente precedente).

Se realizó un análisis descriptivo de todas las variables recogidas. La probabilidad de progresión se estimó utilizando la metodología de Kaplan-Meier. Para testar la existencia de diferencias significativas entre grupos se utilizó el test de rangos logarítmicos. Los factores pronósticos que se incluyeron en la probabilidad de progresión han sido evaluados mediante modelos de riesgo proporcional de Cox.

Se consideraron significativos valores de $\mathrm{P}<$ 0,05. El análisis estadístico se ha realizado con el paquete estadístico SPSS 11,5 para Windows.

\section{RESULTADOS}

Se estudiaron 128 pacientes, que fueron sometidos a linfadenectomía iliaco-obturatriz, en el periodo comprendido entre noviembre de 1995 y julio del 2003, como método de estadiaje para una adecuada selección, con vistas a la realización de un tratamiento local de radioterapia externa.

17 de los $128(13,3 \%)$ pacientes presentaban afectación linfática tumoral en el estudio histológico, motivo por el que se descartó tratamiento radioterapéutico y se inició hormonoterapia. Este grupo de pacientes con afectación ganglionar tenían una edad media de 69,41 (rango 63-78), un tacto rectal anormal en el 94,12\%, una media de PSA de $51,1 \mathrm{ng} / \mathrm{ml}$ (con un rango entre 4,1 y 199), un Gleason mayor o igual a 7 en el 76,47\%, un porcentaje medio de cilindros positivos de $77,50 \%$ y un estadio clínico T3 en el $62,5 \%$ de los casos.

La existencia de una importante diferencia de casos entre ambos grupos ha motivado que no se haya procedido al análisis de las variables implicadas, para poder predecir la afectación linfática.

La estadística descriptiva de los pacientes con linfadenectomía negativa se recoge en la Tabla 1;

Tabla 1

Características generales de los pacientes estudiados.

\begin{tabular}{|c|c|c|c|c|c|c|c|}
\hline & $\mathbf{n}$ & $\%$ & Media & Desviación típica & Mediana & Mínimo & Máximo \\
\hline Edad & 111 & & 70 & 5 & 70 & 55 & 81 \\
\hline $\begin{array}{l}\text { Tacto rectal } \\
\text { Normal } \\
\text { Anormal }\end{array}$ & $\begin{array}{l}25 \\
85\end{array}$ & $\begin{array}{l}22,7 \\
77,3\end{array}$ & & & & & \\
\hline $\begin{array}{l}\text { PSA total } \\
\qquad 20 \\
\quad 20-30 \\
30-50 \\
>50\end{array}$ & $\begin{array}{l}62 \\
15 \\
15 \\
17\end{array}$ & $\begin{array}{l}56,9 \\
13,8 \\
13,8 \\
15,6\end{array}$ & 33,82 & 46,33 & 18 & 2,50 & 300 \\
\hline Volumen prostático & & & & & & & \\
\hline $\begin{array}{l}\text { Gleason preoperatorio } \\
\quad<7 \\
\quad 7 \\
\quad 8 \\
\quad>8\end{array}$ & $\begin{array}{l}24 \\
45 \\
24 \\
16\end{array}$ & $\begin{array}{l}22,0 \\
41,3 \\
22,0 \\
14,7\end{array}$ & 7 & 1 & 7 & 1 & 10 \\
\hline$\%$ cilindros positivos & & & 71,62 & 25,96 & 70 & 10 & 100 \\
\hline Estancia hospitalaria & & & 2,88 & 1,20 & 3 & 1 & 7 \\
\hline $\begin{array}{r}\text { Estadio } \\
\text { T2a } \\
\text { T2b } \\
\text { T3a } \\
\text { T3b }\end{array}$ & $\begin{array}{c}3 \\
41 \\
46 \\
18\end{array}$ & $\begin{array}{c}2,8 \\
38,0 \\
42,6 \\
16,7\end{array}$ & & & & & \\
\hline $\begin{array}{l}\text { Dosis radioterapia } \\
\text { Progresión } \\
\text { Exitus }\end{array}$ & $\begin{array}{l}21 \\
10\end{array}$ & $\begin{array}{c}18,9 \\
9,3\end{array}$ & 66 & 4 & 66 & 45 & 7 \\
\hline
\end{tabular}


éstos fueron seleccionados para realizar tratamiento radioterápico con fuente externa. Los datos más reseñables de esta población son: edad media de 70 años (55-81); predominio de exploración rectal anómala $(77,3 \%)$; el nivel de PSA fue inferior a 20 en el 56,9\%, entre 20-30 en el $13,8 \%$, entre 30 y 50 en el $13,8 \%$ y mayor a 50 en el 15,6\%; el $40 \%$ presentaron un grado de Gleason combinado igual o mayor a 8 en la biopsia transrectal y el $41,3 \%$ correspondieron a un Gleason 7; la media de cilindros positivos fue de $71,62 \%$ con una D.T. de $25,96 \%$; en cuanto a la distribución por estadios clínicos, el 40,8\% correspondieron a un T2 y el 59,2\% a T3.

Según se aprecia en la gráfica de KaplanMeier (Fig. 1), la probabilidad global de no progresión en los pacientes sometidos a radioterapia externa, se fija en el $50 \%$ a 60 meses.

Para establecer posibles marcadores de riesgo, realizamos un análisis más detallado de la probabilidad de no progresión tras radioterapia, realizando una estratificación de la población en base a los factores de riesgo conocidos (nivel de PSA, Gleason de la biopsia, \% de cilindros positivos y estadio clínico) y efectuamos un análisis univariado, cuyos resultados se pueden apreciar en la Tabla 2; en ella podemos ver que tras ajustar en un modelo de regresión de Cox por las variables anteriores ninguna de ellas tiene un efecto significativo sobre la probabilidad de progresión, lo que atribuimos al tamaño muestral y a la magnitud de la diferencia que se deseamos detectar. Sin embargo, se aprecia una diferencia de magnitud considerable, que casi alcanza el valor de $\mathrm{p}$ necesario para considerarlo significativo, en la estratificación según el nivel de PSA en menor o mayor de 20.

Otro de los aspectos que se apuntan y que aparece reflejado gráficamente en la Figura 2 es la probabilidad de no progresión en función de la dosis de Rt recibida, apreciando una disminución de la probabilidad de progresión en aquellos que reciben dosis más altas (mayor o igual a $70 \mathrm{~Gy}$ ).

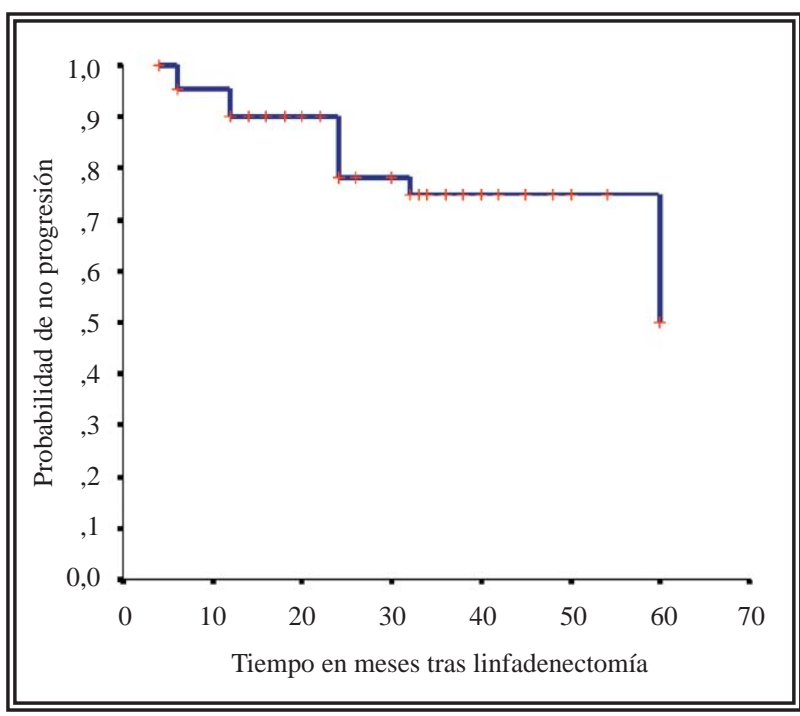

FIGURA 1. Probabilidad de no progresión en pacientes sometidos a linfadenectomia.

En la Tabla 3 se recogen las complicaciones producidas por el tratamiento según la escala de valoración RTOG. Pudiendo apreciar que la mayor parte de las complicaciones son de grado menor, pero que existen 4 inmediatas y 5 tardías mayores. Destaca la aparición tardía de hematuria macroscópica en 4 pacientes siendo de grado mayor en 2 de ellos.

\section{DISCUSIÓN}

La selección apropiada de una opción terapéutica en el cáncer de próstata permanece como

\section{Tabla 2}

Probabilidad de no progresión tras linfadenectomía.

\begin{tabular}{lrrrrr}
\hline Variable & $\mathbf{6}$ meses & $\mathbf{1}$ año & $\mathbf{2}$ años & $\mathbf{5}$ años & $\mathbf{p}$ \\
& & & & & \\
\hline & $95,45 \%$ & $89,95 \%$ & $78,15 \%$ & $49,93 \%$ & \\
$\begin{array}{l}\text { PSA total } \\
\quad<\mathbf{2 0}\end{array}$ & $96,77 \%$ & $93,49 \%$ & $87,46 \%$ & $80,73 \%$ & \\
$\quad>\mathbf{2 0}$ & $93,48 \%$ & $84,78 \%$ & $66,61 \%$ & $44,41 \%$ & \\
$\begin{array}{l}\text { Gleason de biopsia } \\
\quad \mathbf{7}\end{array}$ & $94,2 \%$ & $89,79 \%$ & $80,58 \%$ & $75,54 \%$ & \\
$\quad>\mathbf{8}$ & $97,44 \%$ & $89,74 \%$ & $71,79 \%$ & $35,90 \%$ & \\
$\quad \begin{array}{l}\text { Estadio patológico } \\
\quad \text { pT2 }\end{array}$ & $97,67 \%$ & $95,29 \%$ & $80,25 \%$ & $80,25 \%$ & \\
$\quad$ pT3 & $95,31 \%$ & $87,50 \%$ & $76,56 \%$ & $48,21 \%$ & \\
\% de cilindros positivos & & & & & 0,511 \\
$\quad<70$ & $93,18 \%$ & $93,18 \%$ & $73,21 \%$ & $0 \%$ & \\
$\quad>70 \%$ & $98,39 \%$ & $88,71 \%$ & $82,99 \%$ & $76,60 \%$ & \\
\hline
\end{tabular}

Tras ajustar en un modelo de regresión de Cox por las variables anteriores ninguna de ellas tiene un efecto significativo sobre la probabilidad de progresión. 


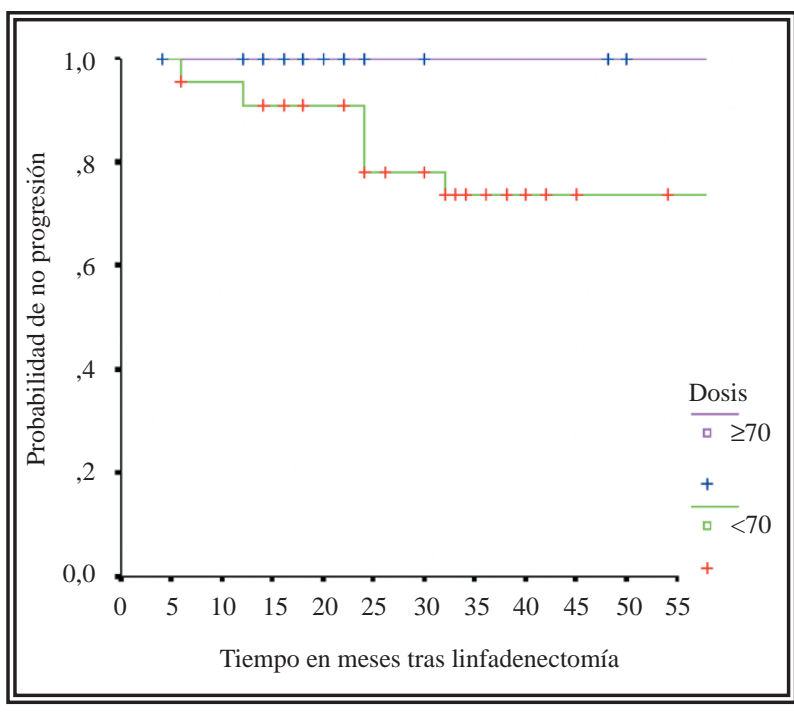

FIGURA 2. Probabilidad de no progresión en pacientes sometidos a linfadenectomía según la dosis de radioterapia recibida.

un aspecto controvertido, ofreciéndose varias alternativas validadas en la literatura para un mismo estadio tumoral, y admitiendo algunos de ellos un abanico más amplio de alternativas que otros. La experiencia de los diversos grupos y la transmitida a través de las casuísticas publicadas son las guías que conducen la elección de una u otra alternativa.

El American College of Surveys en su trabajo "Patterns of Care Survey" en el cáncer de próstata estadio T3, publicó la década de los 90 la estadística de las alternativas terapéuticas elegidas

Tabla 3

Número de efectos secundarios de radioterapia según escala RTOG.

\begin{tabular}{|c|c|c|c|c|c|}
\hline & \multicolumn{2}{|c|}{ Efectos inmediatos } & \multicolumn{2}{|c|}{ Efectos tardios } & \multirow[t]{2}{*}{ Total } \\
\hline & grados $1-2$ & grados 3-4 & grados 1-2 & grados 3-4 & \\
\hline \multicolumn{6}{|l|}{ Genitourinario } \\
\hline Disuria, frecuencia & 8 & 2 & 8 & & 18 \\
\hline Hematuria & & & 2 & 2 & 4 \\
\hline Incontinencia urg & & & 2 & & 2 \\
\hline Dism calibre & 2 & 1 & & & 3 \\
\hline \multicolumn{6}{|l|}{ Gastro-intestinal } \\
\hline Sangrado & 4 & 1 & 3 & 3 & 11 \\
\hline Tenesmo & 2 & & 3 & & 5 \\
\hline \multicolumn{6}{|l|}{ Dolor } \\
\hline Diarrea & 3 & & 2 & & 5 \\
\hline Total & 19 & 4 & 20 & 5 & 48 \\
\hline
\end{tabular}

en este tipo de tumores sobre 3.480 pacientes: Rt sólo (34,3\%) Hormonoterapia sólo $(20,2 \%)$ PR sólo $(15,8 \%)$ tratamiento combinado $(14,1 \%)$.

Antes de la selección de un tratamiento apropiado se genera la necesidad de un adecuado diagnóstico de extensión, que intenta situarnos en la auténtica realidad que afrontamos, $y$, dentro de esta acotación también existen discrepancias sobre los procedimientos que se deben llevar a cabo para proceder al estadiaje tumoral. No cabe duda de que el factor limitante para un tratamiento curativo local es que la enfermedad no esté confinada en la próstata, y, para definir este aspecto la primera estación de las células malignas en su extensión debe de ser nuestra referencia, así pues, debemos dirigir nuestra atención sobre las cadenas iliaco-obturatrices.

Para poder conocer la afectación ganglionar el método gold-standard es la linfadenectomía iliaco-obturatriz, en alguna de sus variantes: clásica, laparoscópica o mediante minilaparotomía; representa un método válido y fiable de estadiaje, como contrapartida a su coste económico por ocupación de quirófano y a su morbilidad (linfoceles, infecciones de herida quirúrgica, tromboembolismos, lesiones del nervio obturador, lesiones de vasos obturadores, edema genital,...) como procedimiento quirúrgico que es, quedando la TAC y la RMN como alternativas menos invasivas pero con mucha menor sensibilidad y especificidad.

La indicación fundamental para proceder al estadiaje ganglionar es que el resultado del mismo vaya a modificar nuestra actuación terapéutica, lo cual, según los nomogramas de actuación de la NCCN, comprendería cualquier $\mathrm{T} 3-\mathrm{T} 4$ o si la probabilidad de afectación indicada por los nomogramas de predicción, basados en factores predictivos como el score de Gleason, el nivel de PSA, el tacto rectal o el volumen tumoral, es mayor del $20 \%{ }^{22}$. Una de las pocas asociaciones internacionales que ha publicado unas guías de consenso 
para el tratamiento del cáncer de próstata en base a la opinión de reputados expertos es la NCCN.Y en tumores de alto riesgo (T3a-T3b,G 810, PSA $>20$ ) las alternativas que se plantean para aquellos individuos con una esperanza de vida mayor a 5 años son: hormonoterapia + radioterapia (acuerdo unánime de expertos), la hormonoterapia aislada o la prostatectomía radical. Y si existiese afectación ganglionar se excluiría la cirugía radical.

La linfadenectomía de estadiaje debe de tener la extensión apropiada, comprendiendo la región entre ambas ramas iliacas desde la bifurcación aórtica hasta el arco púbico. Varios trabajos remarcan la importancia de comprender la cadena hipogástrica y de hacer una linfadenectomía extensiva (iliaca común, externa, interna, obturatriz y presacros) en la cirugía de estadificación. Además se podría completar el estadiaje mediante empleo de técnicas moleculares en el análisis del tejido linfático ${ }^{4,6,16-18}$. Nuestra técnica de linfadenectomía comprende fundamentalmente la cadena iliaco-obturatriz en los limites delimitados por iliaca externa, iliaca interna, alcanzando en la disección ascendente el límite de la bifurcación iliaca y comenzando el extremo caudal a nivel del pubis.

Las proposiciones de cara a evitar estadiajes innecesarios basándose en factores clínicos predictivos de afectación varían en cuanto a las delimitaciones empleadas, variando el porcentaje de positividad que se acepta perder en el diagnóstico; como ejemplo, en las clínicas urológicas de Norteamérica, Link lo fija en un 4\% basándose en las tablas de Partin para la predicción de la afectación ganglionar. El límite superior que los alberga en su mayoría es el delimitado por: PSA $>20$ o Gleason $>$ a 7 o estadio clínico > T2b (AJCC 1997), poniéndose en muchos de ellos limites menos amplios $6,7,10,11,13,19$.

Es importante resaltar que no se han detectado diferencias estadísticamente significativas en lo que a efectos secundarios se refiere entre pacientes sometidos a linfadenectomía previa con respecto a los que no se sometieron a dicha intervención antes de recibir radioterapia externa ${ }^{3}$. Un aspecto controvertido es el empleo en pacientes con afectación ganglionar, existiendo algunas series en las que se incluyen, pero que no vamos a abordar en la presente revisión.
La RTOG publicó su experiencia con más de 1.500 pacientes seguidos durante 15 años, recogidos de 4 ensayos fase III, tratados con Rt únicamente (recibiendo en su mayor parte más de 70 gy), en cánceres de próstata localmente avanzados (fund T3 y T1-T2 con afectación ganglionar) en la era pre-PSA. Sugiriendo una alta probabilidad de supervivencia global a 10 años, en torno al 40\%, y remarcando como factores determinantes para la misma el grado de diferenciación tumoral y el estadio histológico en segundo lugar $^{23}$.

En las series clásicas, en las que se empleó RT externa únicamente en tumores estadio T3-T4, se recogia una supervivencia libre de enfermedad a 10 años que oscila entre el 28\% y el $44 \%$.

Estas series emplean en su mayoría para la detección de la recidiva valoraciones histológicas, de complicada interpretación, o clínicas, que difícilmente se pueden comparar con las series actuales debido a las valoraciones bioquímicas posteriormente introducidas y a la dificultad para su interpretación basándonos en los conocimientos actuales. Se asume por la comunidad científica que estos puntos clásicos de valoración clínica o histológica para la recidiva ofrecen mejores resultados de lo que ofrecerían si se emplease la valoración bioquímica, cifrando esta discordancia en torno al 20\% en las valoraciones de la RTOG. Es por ello que las supervivencias libre de enfermedad no resultan válidas en la equiparación ${ }^{24}$.

Los efectos beneficiosos de la escalada de dosis fueron demostrados en un ensayo randomizado, en el que se comparó el empleo de 64 70 frente al de 78 Gy advirtiendo un aumento significativo de supervivencia libre de enfermedad, que beneficia de manera especial a aquellos con un PSA pretratamiento > a 10 aunque también se aprecia para los que tienen PSA < 10 entre los 64 y los $70 \mathrm{~Gy}$; el precio a pagar consiste en un aumento en el número de complicaciones, fundamentalmente por la mayor afectación rectal ${ }^{25,26}$.

Nuevas alternativas han aparecido para recoger el beneficio de la escalada de dosis sin aumentar el número de complicaciones. La principal variante ha sido la introducción de la radioterapia conformada tridimensional, con una mejor selección del campo de radiación. Brundage realizó un metanálisis de 4 ensayos randomizados que comparan 
la RDT conformada tridimensional frente a la clásica, y se concluye de los resultados obtenidos en estos estudios que la RT 3d: produce menos morbilidad aguda y tardía, parece más eficaz que la clásica y no incrementa la toxicidad a pesar de aumentar la dosis. Todo lo cual se explica por la concentración más adecuada de la dosis sobre el tejido a tratar. En nuestra serie hemos adoptado esta técnica desde el 2002 pero no hemos realizado un análisis comparativo por la importante desigualdad en cuanto a tamaño de ambos grupos ${ }^{27}$.

Desde los años 80 la RTOG ha estudiado el valor potencial del tratamiento hormonal sumado al tratamiento convencional de radioterapia, así ha seguido una linea de investigación que comenzó con el uso de derivados estrogénicos en el ensayo RTOG 8307; posteriormente, y a lo largo de las dos últimas décadas se han ido comunicando trabajos por diversas entidades en los que se ha valorado el papel de los agonistas de la LHRH y de los antiandrógenos no esteroideos en relación con el tratamiento radioterapéutico.

Se comenzó con la evaluación del tratamiento hormonal neoadyuvante y simultáneo. Las hipótesis de trabajo de estos ensayos eran fundamentalmente cuatro:

1. Reducción de volumen (citoreducción-clones andrógeno dependientes): en más del 90\% casos. Se ha cuantificado la misma en estudios experimentales en torno al 37\% a 3 meses. Que según demostró Henderson debe de ser realizado mediante el empleo de agonistas de LHRH pues con antiandrógenos no se alcanza ese grado de reducción.

2. Mecanismo sinérgico de facilitación apoptosis.

3. Disminución de la hipoxia en el interior del tumor, facilitando la acción de la RT.

4. Cobertura de micrometástasis ocultas que se escapan de un tratamiento local pero que según su nivel de andrógeno sensibilidad se pueden ver afectados por un ambiente de deprivación androgénica $^{28-31}$.

En estos ensayos (RTOG 8519, RTOG 8610, RTOG 9413, RTOG 9408, canadienses) se apreciaron las bondades del tratamiento neoadyuvante, apreciándose una mejoría significativa en los grupos con tratamiento hormonal respecto al con- trol local de la enfermedad a 5 años, el tiempo hasta la aparición de eventos metastásicos, control a distancia a los 5 años y respecto al tiempo libre de recidiva bioquímica, en aquellos más recientes que valoraron este parámetro libre. No se encontró una significativa diferencia en la supervivencia global, pero en el 8610 se apreció que al estratificar por el grado de Gleason aquellos con un grado menor a 6 sí presentaban una mejoría significativa de la supervivencia.

Estos ensayos se realizaron sobre poblaciones con tumores localmente avanzados, con grandes volúmenes tumorales o con afectación ganglionar. El siguiente paso ha sido el diseño de ensayos centrados sobre tumores localizados en estadios precoces para valorar si se mantiene el beneficio, y como se ha podido ver en la serie presentada por D'amico, en la que se administró al grupo de estudio supresión androgénica neoadyuvante - simultánea y adyuvante de dos meses, se mantiene el beneficio. Los ensayos RTOG 9910 y Calgb, en marcha, valoran este aspecto particular.

Para precisar la importancia de la temporalidad del tratamiento hormonal respecto al radioterapéutico se llevó a cabo el estudio RTOG 9413 que comparó el tratamiento ablativo androgénico 2 meses antes y durante la RT con la administración del mismo durante un idéntico periodo de tiempo (4 meses) iniciándolo inmediatamente después de finalizar la RT; comprobándose que el intervalo libre de progresión a 4 años era significativamente superior en el grupo de neoadyuvancia, apoyando, con estos resultados, las teorías que hipotetizan la potenciación de efectos del tratamiento con RT en aquellos pacientes que reciben previamente bloqueo hormonal ${ }^{32-41}$.

En la misma línea de establecimiento de tiempos, se ha trabajado para poder definir la extensión recomendable del tratamiento neoadyuvante, se diseñó un ensayo multicéntrico canadiense, en el que, se randomizaron los pacientes para la aplicación del tratamiento supresor androgénico neoadyuvante durante 3 meses en un grupo y 8 meses en el otro. Obteniendo como resultado una ausencia de diferencias significativas en cuanto al intervalo libre de enfermedad a 3 y 5 años, aunque se apreció una tendencia de beneficio en aquellos tumores de alto riesgo ${ }^{42}$. 
Paralelamente al desarrollo del conocimiento en el área de la hormonoterapia previa, se han ido desarrollando ensayos que valoran el aporte que supone el tratamiento adyuvante, bajo la racionalidad de alcanzar un mejor control de la enfermedad en aquellos casos en los que por los factores predictivos que los configuran se espera un peor pronóstico.

Tras unos dubitativos comienzos en esta senda de investigación, con los trabajos RTOG 8610 (2 meses adyuvancia), RTOG 8531 (mantenida HT adyuvante), en los que no se apreciaba diferencia estadísticamente significativa respecto a la supervivencia global (excepto en el subgrupo de tumores Gleason > 7 en Ht mantenida), aunque sí respecto al intervalo libre de recidiva $y$ metástasis.

En 2002 ve la luz el ensayo EORTC 22863 realizado en pacientes con tumores localmente avanzados o mal diferenciados, que fueron asignados aleatoriamente a dos grupos, uno que recibió únicamente RT y otro al que se le realizó tratamiento complementario con bloqueo androgénico durante 3 años empezando desde el inicio de la RT. Los resultados ofrecieron significación estadística para: mejor control loco-regional de la enfermedad (98\% vs 84\%), prolongación del intervalo hasta la aparición de metástasis $(90 \%$ vs $71 \%$, prolongación del intervalo libre de enfermedad (74\% vs 40\%), prolongación del tiempo hasta la recidiva bioquímica (76\% vs $45 \%$ ) y aumento del tiempo de supervivencia global a 5 años (78\% vs 62\%), en aquellos pacientes que recibieron tratamiento adyuvante.

Apreciándose por estos estudios las bondades del tratamiento adyuvante, queda por definir la duración apropiada de dicho tratamiento, que es objeto de estudio en el ensayo EORTC 22961, cuyo diseño confronta 2,5 años frente a 6 meses de tratamiento adyuvante ${ }^{43-49}$.

Como contrapunto a esta beneficiosa actividad se sitúan los secundarismos que de dicho tratamiento se derivan. De el EORTC 22863 se concluye que los factores relacionados con un aumento de las complicaciones generadas por la RT son: dosis de radiación, edad, cirugía abdominal previa; pero no influye la combinación de tratamiento hormonal, lo que, es consistente con otras valoraciones como la del RTOG 94-06. Los tratamientos hormonales mantienen la morbilidad que les es propia: anemia, osteoporosis, pérdida de vigor, ginecomastia,.... Pero no potencian los efectos secundarios de la RT.

Tampoco se ha apreciado que la hormonoterapia adyuvante interfiera con la respuesta a un tratamiento hormonal de salvataje después de una recaída post-RT.

Los efectos de estos estudios no se han hecho esperar y en los Patterns of Care survey for prostate cancer de 1999 ya se recoge un cambio en el porcentaje de pacientes con cáncer de próstata tratados mediante asociación de deprivación androgénica, que pasa del 8\% en 1994 al 51\% de 1999.

\section{CONCLUSIONES}

El tratamiento de pacientes de alto riesgo de progresión como el que enfrentamos en los cánceres de próstata avanzados con factores clínicos pronósticos adversos, puede ser afrontado mediante el empleo de radioterapia externa sin importante menoscabo de su calidad de vida, pues, produce un índice bajo de secundarismos específicos. La radioterapia de conformación 3D ayuda a reducir los mismos.

El empleo de dosis más altas de radioterapia parece más eficaz en cuanto al control de la enfermedad, pero, conlleva un aumento de los efectos secundarios, por lo que sólo con las técnicas 3D podrá llegar a realizarse con cierta seguridad.

Los estudios clínicos realizados en el terreno de la hormonoterapia neoadyuvante demuestran aumento del control de la enfermedad, apreciándose en algunos de ellos aumento de la supervivencia global. Debe de realizarse con análogos de la LH-RH y en cuanto a su duración no parece que regimenes superiores a los tres meses aporten beneficio adicional alguno.

También se ha demostrado la bondad del tratamiento adyuvante con respecto al control de la enfermedad, y, sólo queda por definir cuál debe de ser la duración óptima de dicho tratamiento, para lo cual ya se están realizando investigaciones.

Aunque no hemos obtenido un resultado estadísticamente significativo, parece que el factor clínico más implicado en el pronóstico de estos pacientes es el nivel de PSA total. 


\section{REFERENCIAS}

1. Herranz Amo F, Arias Funez F, Arrizabalaga Moreno M, Calahorra Fernandez FJ, Carballido Rodríguez J, Diz Rodríguez R et al. El cáncer de próstata en la comunidad de Madrid en el 2000 I.- Incidencia. Actas Urol Esp. 2003 May;27(5):323-334.

2. Prostate Cancer (2005) (update) ISBN 90-70244-29-2. European urology guidelines.

3. Greskovich FJ, Zagers GK, Sherman NE, Johnson DE. Complications following external beam radiation therapy for prostate cancer: an analysis of patients treated with and without staging pelvic lymphadenectomy. J. Urol. 1991 Sep;146(3):798-802.

4. Brenot-Rossi I, Bastide C, Garcia S, Dumas S, Stemi B, Pasquier $J$ et al. Limited pelvic lymphadenectomy using the sentinel lymph node procedure in patients with localized prostate carcinoma: a pilot study. Brenot. Eur J Nucl Med Mol Imaging. 2005 Jun;32(6):635-640.

5. Burkhard FC, Schumacher M, Thalmann GN and Studer U.E. Is pelvic lymphadenectomy really necessary in patients with a serum prostate specific antigen level of $<10$ $\mathrm{ng} / \mathrm{ml}$ undergoing radical prostatectomy for prostate cancer ? BJU Int. 2005Feb;95(3):275-278.

6. Heidenreich A, Varga $Z$ and Von Knobloch R. Extended pelvic lymphadenectomy in patients undergoing radical prostatectomy: high incidence of lymph node metastasis. J Urol. 2002 Apr;167(4):1681-1686.

7. Burkhard FC, Bader P, Schneider E, Markwalder R., Studer U.E. Reliability of preoperative values to determine the need for lymphadenectomy in patients with prostate cancer and meticulous lymph node disection. Eur Urol. 2002 Aug;42(2):84-90.

8. Narayan P, Fournier G, Gajendran V, Leidich R, Lo R., Wolf JS Jr et al. Utility of preoperative serum prostate-specific antigen concentration and biopsy Gleason score in predicting the risk of lymph node metastasis in prostate cancer. Urology. 1994 Oct;44(4):519-524.

9. Clark T, Parekh DJ, Cookson MS, Chang SS, Smith ER Jr, Wells $\mathrm{N}$ et al. Randomized prospective evaluation of extended versus limited lymph node disecction in patients with clinically localized prostate cancer. J Urol. 2003 Jan;169 (1): 145-147.

10. Link RE, Morton RA. Indications for pelvic lymphadenectomy in prostate cancer. Urol Clin North Am. 2001 Aug; 28(3):491-498.

11. Hattori R, Kinukawa T, Ono Y, Kato N, Yamada S, Takeda A et al. Laparoscopic pelvic lymphadenectomy for localized prostate cancer. Hinyokika Kiyo 1996 Oct;42(10):775-780.

12. Peneau M, Villers A, Molinie V, Theis D and Soulie M. Indications for pelvic lymphadenectomy in clinically localized prostate cancer. Prog Urol. 2004 Jun;14(3):287-294.

13. Alagiri M, Colton MD, Seidmon EJ. The staging pelvic lymphadenectomy: implications as an adjunctive procedure for clinically localized prostate cancer. Br J Urol. 1997 Aug;80(2):243-246.

14. Hoenig DM, Chi S, Porter C, Tackett L, Smith DS, Cohen SI et al. Risk of nodal metastases at laparoscopic pelvic lymphadenectomy using PSA, Gleason score and clinical stage in men with localized prostate cancer. Endourol 1997 Aug; 11(4):263-265.

15. Cagiannos I, Karakiewicz P, Eastham JA, Ohori M, Rabbani F, Gerigk C et al. A preoperative nomogram identififying decreased risk of positive pelvic lymph nodes in patients with prostate cancer. J Urol. 2003 Nov;170(5): 1798-1803.
16. Edelstein RA, Zietman AL, de las Morenas A, Krane RJ, Babayan RK, Dallow KC et al. Implications of prostate micro metastases in pelvic lymph nodes. an archival tissue study. Urology. 1996 Mar;47(3):370-375.

17. Allaf ME, Palapattu GS, Trock BJ, Carter HB, Walsh PK. Anatomical extent of lymph node dissection: impact on men with clinically localized prostate cancer. J Urol. 2004 Nov; 172 (5 Pt 1):1840-1844.

18. Bader P, Burkhard FC, Markwalder R, Studer UE. Is a limited lymph node disecction an adequate staging procedure for prostate cancer. J Urol. 2002 Aug;168(2):514-518.

19. El-Galley RE, Keane TE, Petros JA, Sanders WH, Clarke HS, Cotsonis GA et al.Evaluation of staging lymphadenectomy in prostate cancer. Urology 1998 Oct;52(4):663-667.

20. Waehre H, Wanderaas EH, Paus E, Fossa SD. Prediction of lymph node metastases by a prostate-specific antigen and prostatic acid phosphatase in clinical T3/T4 M0 prostatic cancer. Eur Urol. 1992;22(1):33-38.

21. Steiner MS, Marshall FF. Mini-laparotomy staging pelvic lymphadenectomy (minilap). Alternative to standard and laparoscopic pelvic lymphadenectomy. Urology. 1993 Mar; 41(3):201-206.

22. Douglas Scherr, Peter W. Swindle, Peter T. Scardino. National comprehensive cancer network guidelines for the management of prostate cancer. Urology. 2003;61(Suppl $\left.2^{\mathrm{a}}\right)$ :14-24.

23. Roach M 3rd, Lu J, Pilepich MV, Asbell SO, Mohiuddin M, Terry $\mathrm{R}$ et al. Long-term survival after radiotherapy alone: radiation therapy oncology group prostate cancer trials. J Urol. 1999 March;161(3):864-868.

24. Iczkowski KA, Bostwick DG. El efecto de la radioterapia en la histopatología de la próstata y la determinación del cáncer residual. Rev Urol. 2002;3(4):139-146.

25. Kuban D, Pollack A, Huang E, Levy L, Long L, Starkschall $\mathrm{G}$ et al. Hazards of dose escalation in prostate cancer radiotherapy. Int $\mathrm{J}$ Radiat Oncol Biol Phys. 2003 Dec; 57(5):1260-1268.

26. Pollack A, Zagars GK, Starkschall G, Antolak JA, Lee JJ, Huang $\mathrm{E}$ et al. Postate cancer radiation dose response : results of the MD Anderson phase III randomized trial. Int J Radiat Oncol Biol Phys. 2002 Aug;53(5):1097-1105.

27. Brundage M, Lukka H, Crook J, Warde P, Bauman G, Catton $\mathrm{C}$ et al. The use of conformal radiotherapy and the selection of radiation dose in $\mathrm{T} 1$ or $\mathrm{T} 2$ low or intermediate risk prostate cancer - a sistematic revue. Radiother Oncol. 2002 Sep;64(3):239-250.

28. Akakura K, Bruchovsky N, Goldenberg S1, Renie PS, Buckley AR, Sullivan RD. Effects of intermittent androgen suppression on androgen-dependent tumors. Apoptosis and serum prostate specific antigen. Cancer. 1993;71: 2782-2790

30. Sneller ZW, Hop WC, Carpentier PJ, Schroeder FH. Prognosis and prostatic volume changes during endocrine management of prostate cancer: a longitudinal study. J Urol. 1992 Mar;147(3Pt2):962-966.

30. Hara I, Miyake H, Yamada Y, Takechi Y. Neoadjuvant androgen withdrawal prior to external radiotherapy for locally advanced adenocarcinoma of the prostate. Int $\mathrm{J}$ Urol. 2002 Jun;9(6):322-328; discussion 328.

31. Zietman AL, Nakfoor BM, Prince EA, Gerweck LE. The effect of androgen deprivation and radiation therapy on an androgensensitive murine tumor: an in vitro and in vivo study. Cancer J Sci Am. 1997;3:31-36. 
32. Stock RG, Cahlon O, Cesaretti JA, Kollmeier MA, Stone NN. Combined modality treatment in the management of high-risk prostate cancer. Int J Radiat Oncol Biol Phys. 2004 Aug;59(5):1352-1359.

33. Pilepich M V, Winter K, John MJ. Phase III Radiation Therapy Oncology Group (RTOG) trial 86-10 of androgen deprivation adjuvant to definitive radiotherapy in locally advanced carcinoma of the prostate. Int $\mathrm{J}$ Radiat Oncol Biol Phys. 2001;50:1243-1252.

34. Laverdiére J, Gomez JL, Cusan L, Suburu ER, Diamond P, Lemay $\mathrm{M}$ et al. Beneficial effect of combination hormonal therapy administered prior and following external beam radiation therapy in localized prostate cancer. Int $\mathrm{J}$ Radiat Oncol Biol Phys. 1997;37(2):247-252.

35. Roach M III, De Silvio M, Lawton C, Uhl V, Machtay M, Seider MJ et al. Phase III trial comparing whole-pelvic versus prostate-only radiotherapy and neoadjuvant versus adjuvant combined androgen suppression. Radiation Therapy Oncology Group 9413. J Clin Oncol. 2003;21(10): 1904-1911.

36. Lawton CA, Winter K, Byhardt R.,Sause WT, Hanks GE, Russell AH et al. Androgen suppression plus radiation versus radiation alone for patients with D1 (pn+) adenocarcinoma of the prostate (results based on a national prospective randomized trial, RTOG 85-31) Int J Radiat Oncol Biol Phys. 1997;38(5):931-939.

37. D’Amico AV, Manola J, Loffredo M, Renshaw AA, DellaCroce A, Kantoff PW. 6-month androgen suppression plus radiation therapy vs radiation therapy alone for patients with clinically localized prostate cancer. JAMA 2004 Aug;292(7):821-827.

38. Zietman AL, Prince EA, Bruce M, Shipley WU. Neoadyuvant androgen suppression with radiation in the management of locally advanced adenocarcinoma of the prostate: experimental and clinical results. Urology. 1997 March;49(suppl 3a):74-83.

39. Pisansky TM. Use of neoadyuvant and adyuvant therapy to prevent or delay recurrence of prostate cancer in patients undergoing radiation treatment for prostate cancer. Urology. 2003 Aug;62(Suppl 6b):36-45.

40. Pilepich MV, Krall JM, Al-Sarraf M, John MJ, Doggett RL, Sause WT et al. Androgen deprivation with radiation therapy compared with radiation therapy alone for locally advanced prostatic carcinoma : a randomized comparative trial of the radiation therapy oncology group. Urology 1995 Apr;45(4):616-623.

41. Ryan CJ, Zelefsky MJ, Heller G, Regan K, Leibel SA, Scher HI et al. Five-year outcomes after neoadjuvant chemotherapy and conformal radiotherapy in patients with high-risk localized prostate cancer. Urology. 2004 Jul;64(1):90-94.

42. Crook J, Ludgate C, Malone S, Lim J, Perry G, Eapen L et al. Report of a multicenter Canadian phase III randomized trial of 3 months vs. 8 months neoadjuvant androgen deprivation before standard-dose radiotherapy for clinically localized prostate cancer. Int $\mathrm{J}$ Radiat Oncol Biol Phys. 2004 Sep;1;60(1):15-23.
43. Schmidt JD, Gibbons RP, Murphy GP and Bartolucci A. Adyuvant therapy for localized prostate cancer. Cancer. 1993;71(3 suppl):1005-1013.

44. Lawton CA, Winter K, Murray K, Machtay M, Mesic JB, Hanks GE et al. Updated results of the phase III Radiation Therapy Oncology Group (RTOG) trial 85-31 evaluating the potential benefit of androgen suppression following standard radiation therapy for unfavorable prognosis carcinoma of the prostate. Int $J$ Radiat Oncol Biol Phvs. 2001;49(4):937-946.

45. Bolla M, Collette L, Blank L, Warde P, Dubois JB, Mirimanoff $\mathrm{RO}$ et al. Long-term results with inmediate androgen supression and external irradiation in patients with locally advanced prostate cancer (an EORTC study): a phase III randomized trial. Lancet. 2002;360(9327):103106.

46. Bolla M. Adjuvant hormonal treatment with radiotherapy for locally advanced prostate cancer. Eur Urol. 1999;35 (suppl 1):23-26.

47. D'Amico A.V. Radiation and hormonal therapy for locally advanced and clinically localized prostate cancer. Urology 2002;Sept;60(Suppl 3a):32-38.

48. Hanks GE, Pajak TF, Porter A,Grignon D, Brereton H, Venkatesan V et al. Phase III trial of long-term adjuvant androgen deprivation after neoadjuvant hormonal cytoreduction and radiotherapy in locally advanced carcinoma of the prostate: the Radiation Therapy Oncology Group Protocol 92-02; Radiation Therapy Oncology Group. J Clin Oncol. 2003;Nov 1;21(21):3972-3978.

49. Laverdiere J, Nabid A, De Bedoya LD. The efficacy and sequencing of a short course of androgen suppression on freedom from biochemical failure when administered with radiation therapy for T2-T3 prostate cancer. J Urol. 2004 Mar;171(3):1137-1140.

50. Valicenti RK, Winter K, Cox JD, Sandler HM, Bosch W, Vijayakumar S et al. RTOG 94-06 : Is the addition of neoadyuvant hormonal therapy to dose-escalated $3 \mathrm{~d}$ conformal radiation therapy for prostate cancer associated with treatment toxicity? Int J Radiat Oncol Biol Phys. 2003 Nov; 57(3):614-620.

51. Shipley WU, Lu JD, Pilepich MV, Heydon K, Roach M, Wolkov HB et al. Effect of a short course of neoadyuvant hormonal therapy on the response to subsequent androgen supresión in prostate cancer patients with relapse alter radiotherapy: a secondary análisis of the randomized protocol RTOG 86-10. RTOG 86-10 Int J Radiat Oncol Biol Phys. 2002 Dec;54(5):1302-1310.

Dr. A. Barbagelata López

Complejo Hospitalario Juan Canalejo

Xubias de Arriba, 84

15006 La Coruña

E-mail: barbage2004@yahoo.com

(Trabajo recibido el 18 de abril de 2006) 